\title{
ONE RADIUS THEOREM FOR THE EIGENFUNCTIONS OF THE INVARIANT LAPLACIAN
}

\author{
E. G. KWON
}

(Communicated by Clifford J. Earle, Jr.)

\begin{abstract}
Let $B$ be the open unit ball in $C^{n}$ with its boundary $S$. Suppose that $\alpha \geq \frac{1}{2}$ and $u(z)=\left(1-|z|^{2}\right)^{n(1-\alpha)} F(z)$ for some $F(z) \in C(\bar{B})$. If for every $z \in B$ there corresponds an $r(z): 0<r(z)<1$ and an automorphism $\psi_{z}$ with $\psi_{z}(0)=z$ such that

$$
u(z)=\frac{1}{g_{\alpha}(r(z))} \int_{S} u \circ \psi_{z}(r(z) \zeta) d \sigma(\zeta),
$$

then $\widetilde{\Delta} u(z)=-4 n^{2} \alpha(1-\alpha) u(z), z \in B$. Here $\widetilde{\Delta}$ is the invariant Laplacian and $g_{\alpha}(r)$ is the hypergeometric function $F\left(n-n \alpha, n-n \alpha, n ; r^{2}\right)$.
\end{abstract}

\section{INTRODUCTION}

Let $\psi_{z}$ be an automorphism, that is, a one-to-one holomorphic map onto itself, of the open unit ball $B$ of $\mathbf{C}^{n}$ satisfying $\psi_{z}(0)=z$. Let $\phi_{z}$ be one of such an automorphism defined by

$$
\phi_{z}(w)=\frac{z-P_{z} w-s_{z}\left(w-P_{z} w\right)}{1-\langle w, z\rangle}, \quad w \in B,
$$

where $P_{z}$ is the orthogonal projection of $\mathbf{C}^{n}$ onto the subspace generated by $z,\langle w, z\rangle=\sum_{j=1}^{n} w_{j} \bar{z}_{j}, s_{z}=\sqrt{\left(1-|z|^{2}\right)}$, and $|z|^{2}=\langle z, z\rangle$. For $u \in C^{2}(B)$, its invariant Laplacian $\widetilde{\Delta}$ is well defined by

$$
(\tilde{\Delta} u)(z)=\Delta\left(u \circ \psi_{z}\right)(0), \quad z \in B,
$$

where $\Delta$ is the ordinary Laplacian. Our starting point is the following mean value theorem [4, Theorem 4.2.4].

Theorem A. Given a complex number $\lambda$, any function $u(z) \in C^{2}(B)$ satisfying

$$
\widetilde{\Delta} u(z)=\lambda u(z), \quad z \in B,
$$

has mean value property

$$
g_{\alpha}(r) u(z)=\int_{S} u \circ \psi_{z}(r \zeta) d \sigma(\zeta), \quad z \in B, 0 \leq r<1 .
$$

Received by the editors July $25,1990$.

1980 Mathematics Subject Classification (1985 Revision). Primary 31 B35.

Key words and phrases. Invariant Laplacian, one radius property.

The research was partially supported by Yonam Foundation. 
Here and throughout $\lambda$ and $\alpha$ are related to be

$$
\lambda=\lambda_{\alpha}=-4 n^{2} \alpha(1-\alpha),
$$

$d \sigma$ is the normalized rotation invariant positive Borel measure on the boundary $S$ of $B$, and

$$
g_{\alpha}(r)=\int_{S}\left(\frac{1-r^{2}}{\left|1-r \zeta_{1}\right|^{2}}\right)^{n \alpha} d \sigma(\zeta) .
$$

The space of all $u(z)$ satisfying $(1)$ is denoted by $X_{\lambda}$. This space is invariant under automorphism. See [4, Chapter 4] for the terminology and related properties.

When $\lambda=0$, Theorem $\mathrm{A}$ has a strong converse known as the one radius theorem [4, Theorem 4.3.4]. See [2] also for the same vein. Motivated by these, our goal here is in the description of the complex numbers $\alpha$ and the smoothness of functions $u(z)$ that makes the following property valid.

One-radius property for $\alpha$ and $u$. If for every $z \in B$ there corresponds an $r(z): 0<r(z)<1$ such that

$$
g_{\alpha}(r(z)) u(z)=\int_{S} u \circ \phi_{z}(r(z) \zeta) d \sigma(\zeta)
$$

then $u(z) \in X_{\lambda}$.

We denote this property by $\operatorname{RP}(\alpha ; u)$ in short. Rudin's one radius theorem says that $1 \operatorname{RP}(0 ; u)=1 \operatorname{RP}(1 ; u)$ is true if $u(z) \in C(\bar{B})$. Izuchi [1] proved that $1 \operatorname{RP}(1 ; u)$ fails for a bounded real analytic $u(z)$. In this paper, we first confine ourselves to $\operatorname{Re} \alpha>\frac{1}{2}$. Our results say that under our growth condition on $u, \operatorname{RP}(\alpha ; u)$ is true if and only if $\alpha$ is real (Theorem 3 ), and the growth condition on $u$ cannot be improvable (Theorem 2 ). Our last section deals with the case $\alpha=\frac{1}{2}$. All unexplained notations and properties will be referred to [4].

\section{ONE RADIUS THEOREM FOR $X_{\lambda}$}

Lemma 1. Let $\alpha \in \mathrm{C}$. Then

$$
g_{\alpha}(r)=g_{1-\alpha}(r)=\left(1-r^{2}\right)^{n(1-\alpha)} \mathscr{F}(\alpha: r),
$$

where

$$
\mathscr{F}(\alpha: r)=\int_{S} \frac{\left(1-r^{2}\right)^{2 n \alpha-n}}{\left|1-r \zeta_{1}\right|^{2 n \alpha}} d \sigma(\zeta) .
$$

If $\alpha$ is real then $\mathscr{F}(\alpha: r)$ is an increasing function of $r$. If $\alpha>\frac{1}{2}$ then $\mathscr{F}(\alpha: r)$ tends to a finite limit $\mathscr{F}(\alpha)=\lim _{r \rightarrow 1} \mathscr{F}(\alpha: r)$. Also

$$
\mathscr{G}\left(\frac{1}{2}: r\right)=\frac{1}{r^{2}} \log \frac{1}{1-r^{2}} \mathscr{F}\left(\frac{1}{2}: r\right)^{-1}
$$

is a continuous function of $r$ that tends to a finite limit $\mathscr{G}\left(\frac{1}{2}\right)=$ $\lim _{r \rightarrow 1} \mathscr{G}\left(\frac{1}{2}: r\right)$.

Theorem 1. Suppose $\alpha>\frac{1}{2}$ and $u(z)=\left(1-|z|^{2}\right)^{n(1-\alpha)} F(z)$ for some $F(z) \in$ $C(\bar{B})$. If for every $z \in B$ there corresponds an $r(z): 0<r(z)<1$ and an 
automorphism $\psi_{z}$ such that

$$
g_{\alpha}(r(z)) u(z)=\int_{S} u \circ \psi_{z}(r(z) \zeta) d \sigma(\zeta)
$$

then

$$
u(z)=\frac{1}{\mathscr{F}(\alpha)} \int_{S} P^{\alpha}(z, \zeta) F(\zeta) d \sigma(\zeta), \quad z \in B
$$

where $P(z, w)$ denotes the invariant Poisson kernel. In particular, $1 \operatorname{RP}(\alpha ; u)$ is true.

Proof of Lemma 1. By [4, Remark, p. 44],

$$
\begin{aligned}
\mathscr{F}(\alpha: r) & =\int_{S} \frac{\left(1-r^{2}\right)^{2 n \alpha-n}}{\left|1-\left\langle r \eta, \phi_{r \eta}(\zeta)\right\rangle\right|^{2 n \alpha}} P\left(\phi_{r \eta}^{-1}(0), \zeta\right) d \sigma(\zeta) \\
& =\int_{S} \frac{\left(1-r^{2}\right)^{2 n \alpha-n}}{\left|1-\left\langle\phi_{r \eta}(0), \phi_{r \eta}(\zeta)\right\rangle\right|^{2 n \alpha}} \frac{\left(1-r^{2}\right)^{n}}{|1-\langle r \eta, \zeta\rangle|^{2 n}} d \sigma(\zeta),
\end{aligned}
$$

which turns out to be

$$
\int_{S} \frac{d \sigma(\zeta)}{|1-\langle r \eta, \zeta\rangle|^{2 n(1-\alpha)}}
$$

once we use the identity [4, Theorem 2.2 .5$]$

$$
1-\left\langle\psi_{a}(z), \psi_{a}(w)\right\rangle=\frac{\left(1-|a|^{2}\right)(1-\langle z, w\rangle)}{(1-\langle z, a\rangle)(1-\langle a, w\rangle)},
$$

$a \in B, z, w \in \bar{B}$. Hence $\left(1-r^{2}\right)^{n(1-\alpha)} \mathscr{F}(\alpha: r)=g_{1-\alpha}(r)$. That $g_{\alpha}(r)=$ $g_{1-\alpha}(r)$ is noted in [4, Corollary, p. 51]. The second part follows from the subharmonicity of the integrand and [4, Proposition 1.4.10].

Proof of Theorem 1. Let

$$
v(z)=\frac{1}{\mathscr{F}(\alpha)} \int_{S} P^{\alpha}(z, \zeta) F(\zeta) d \sigma(\zeta), \quad z \in B
$$

Then $v(z) \in X_{\lambda}$ because $P^{\alpha}(z, \zeta) \in X_{\lambda}$ [4, Theorem 4.2.2], so that it suffices to show that $h(z) \equiv u(z)-v(z) \equiv 0$. Consider first the function defined by

$$
G(z)= \begin{cases}\left(1-|z|^{2}\right)^{n(\alpha-1)} v(z), & z \in B, \\ F(z), & z \in S .\end{cases}
$$

For $\zeta \in S$,

$$
\begin{aligned}
|G(r \zeta)-G(\zeta)| & \leq\left|G(r \zeta)-\frac{\mathscr{F}(\alpha: r)}{\mathscr{F}(\alpha)} F(\zeta)\right|+|F(\zeta)| \frac{\mathscr{F}(\alpha: r)}{\mathscr{F}(\alpha)}-1 \mid \\
& =|(I)|+|(I I)| .
\end{aligned}
$$

Since $F(z) \in C(S)$, by Lemma $1,(I I) \rightarrow 0$ as $r \rightarrow 1$ uniformly on $S$. Also since

$$
(I)=\frac{1}{\mathscr{F}(\alpha)} \int_{S} \frac{\left(1-r^{2}\right)^{2 n \alpha-n}}{|1-\langle r \zeta, \eta\rangle|^{2 n \alpha}}[F(\eta)-F(\zeta)] d \sigma(\eta),
$$

by dividing $S$ into two parts-a small neighborhood $Q$ of $\zeta$ and $S-Q$-it is routine to see that $(I) \rightarrow 0$ as $r \rightarrow 1$ uniformly on $S$. Therefore $G(z) \in C(\bar{B})$. 
Now the function $H(z)$ defined by

$$
H(z)= \begin{cases}\left(1-|z|^{2}\right)^{n(\alpha-1)} h(z), & z \in B, \\ 0, & z \in S,\end{cases}
$$

is of $C(\bar{B})$. We will show $H \equiv 0$ in $B$. Suppose $|H(z)|>0$ for some $z \in B$. Then the set $E$ on which $|H(z)|$ takes the maximum is compact. We can take $z_{0} \in E$ such that $\operatorname{dist}\left(z_{0}, S\right)=\operatorname{dist}(E, S)$. Since both $u(z)$ and $v(z)$ satisfy (4), we have (4) with $h(z)$ in place of $u(z)$, so that

$$
H(z) g_{\alpha}(r(z))=\int_{S}\left(1-|z|^{2}\right)^{n(\alpha-1)} h \circ \psi_{z}(r(z) \zeta) d \sigma(\zeta), \quad z \in B .
$$

But the right side of $(6)$ is

$$
\int_{S} P^{1-\alpha}(r(z) \zeta, z) H \circ \psi_{z}(r(z) \zeta) d \sigma(\zeta)
$$

once we use the identity (5). If we apply (6) and (7) to $z_{0}$ with its corresponding $r\left(z_{0}\right)=r_{0}$, we obtain, by $(3)$,

$$
\left|H\left(z_{0}\right)\right|=\frac{1}{\mathscr{F}\left(\alpha: r_{0}\right)}\left|\int_{S} \frac{H \circ \psi_{z_{0}}\left(r_{0} \zeta\right)}{\left|1-\left\langle r_{0} z_{0}, \zeta\right\rangle\right|^{2 n(1-\alpha)}} d \sigma(\zeta)\right|,
$$

which is, by (3) once more, strictly less than

$$
\frac{\mathscr{F}\left(\alpha: r_{0}\left|z_{0}\right|\right)}{\mathscr{F}\left(\alpha: r_{0}\right)}\left|H\left(z_{0}\right)\right| \leq\left|H\left(z_{0}\right)\right| .
$$

This contradiction proves our assertion.

\section{ON SMOOTHNESS}

Because of the symmetry, $g_{\alpha}=g_{1-\alpha}$ and $X_{\lambda_{\alpha}}=X_{\lambda_{1-\alpha}}$, we can have the proper substitute for the case $\alpha<\frac{1}{2}$, that is, Theorem 1 with all $\alpha$ replaced by $1-\alpha$. On the other hand, if $\alpha<\frac{1}{2}$ and $\left(1-|z|^{2}\right)^{n(\alpha-1)} u(z)$ can be of $C(\bar{B})$, then we can easily check from Theorem 1 that $1 \mathrm{RP}(\alpha ; u)=1 \operatorname{RP}(1-\alpha ; u)$ is true. But if $\alpha \leq \frac{1}{2}$, there is no $u \in X_{\lambda}, u \neq \equiv 0$ with $\left(1-|z|^{2}\right)^{n(\alpha-1)} u(z)$ bounded. This can be checked by comparing the order of $g_{\alpha}(r)$ as $r \rightarrow 1$ after integrating $\left(1-\left|\psi_{z}(r \zeta)\right|^{2}\right)^{n(\alpha-1)} \mathcal{u} \circ \psi_{z}(r \zeta)$ with respect to $d \sigma(\zeta)$ and using (2) in Theorem A and (5). This fact and Theorem 1 gives the following solution to the boundary value problem.

Corollary. Let $\alpha$ be real and let $f \in C(S), f \not \equiv 0$, be given. Then there is a solution $u(z)$ such that

(i) $\widetilde{\Delta} u(z)=\lambda u(z)$ in $B$,

(ii) with boundary value $f(\zeta),\left(1-|z|^{2}\right)^{n(\alpha-1)} u(z)$ is of $C(\bar{B})$

if and only if $\alpha>\frac{1}{2}$. In fact,

$$
u(z)=\frac{1}{\mathscr{F}(\alpha)} \int_{S} P^{\alpha}(z, \zeta) f(\zeta) d \sigma(\zeta)
$$

is the unique solution when $\alpha>\frac{1}{2}$.

Our proof of Theorem 1 covers the case $\alpha=1$. But in (8) equality occurs only when $\alpha=1$ because $\mathscr{F}(\alpha: r)$ is strictly increasing if $\alpha$ is real and $\alpha \neq 1$. 
So it seems to be that the compactness of $E$ when $\alpha \neq 1$ is not so much crucial compared to the case $\alpha=1$. Nonetheless we can explain by use of an example of Izuchi that our growth condition cannot be weakened.

Theorem 2. For $\alpha>\frac{1}{2}$, there is a function $u(z)$ such that

(i) $u(z) \notin X_{\lambda}$

(ii) $\left(1-|z|^{2}\right)^{n(\alpha-1)} u(z)$ is bounded real analytic in $B$,

(iii) for every $z \in B$, there is a radius $r(z): 0<r(z)<1$ by which $u(z)$ satisfies (4).

Proof. Izuchi [1] constructed a bounded positive radial real analytic function $F(z)$ on $B$ such that

$$
\sup _{\zeta \in S} F \circ \phi_{z}\left(\delta_{j} \zeta\right)<F(z)<\inf _{\zeta \in S} F \circ \phi_{z}\left(\sigma_{j} \zeta\right)
$$

for some $\delta_{j}, \sigma_{j}$ depend on $z$ (see [1, p. 830]). If we fix $\alpha$ and set $u(z)=$ $g_{\alpha}(z) F(z)$, then by (9) and (2),

$$
\int_{S} u \circ \phi_{z}\left(\delta_{j} \zeta\right) d \sigma(\zeta)<F(z) \int_{S} g_{\alpha} \circ \phi_{z}\left(\delta_{j} \zeta\right) d \sigma(\zeta)=u(z) g_{\alpha}\left(\delta_{j}\right)
$$

and

$$
u(z) g_{\alpha}\left(\sigma_{j}\right)=F(z) \int_{S} g_{\alpha} \circ \phi_{z}\left(\sigma_{j} \zeta\right) d \sigma(\zeta)<\int_{S} u \circ \phi_{z}\left(\sigma_{j} \zeta\right) d \sigma(\zeta),
$$

so that by the continuity on $r$ of

$$
\frac{1}{g_{\alpha}(r)} \int_{S} u \circ \phi_{z}(r \zeta) d \sigma(\zeta)
$$

(iii) is valid. This $u(z)$ satisfy (i), because of the strict inequality in (10), and (ii) also, because $F(z)$ and $\left(1-|z|^{2}\right)^{n(\alpha-1)} g_{\alpha}(z)$ are bounded real analytic.

\section{ONE-RADIUS PROPERTY FAILS FOR NONREAL $\alpha$}

Lemma 2. (1) If $\frac{1}{2} \leq a_{1}<a_{2}$ then $g_{a_{1}}(r)<g_{a_{2}}(r), 0<r<1$.

(2) For a given nonreal $\beta$ with $\operatorname{Re} \beta>\frac{1}{2}$, there exist $r$ and a such that (i) $0<r<1$, (ii) $\frac{1}{2}<a<\operatorname{Re} \beta$, and (iii) $g_{a}(r)=g_{\beta}(r)$.

Theorem 3. Let $\operatorname{Re} \alpha>\frac{1}{2}$. Then the following are equivalent:

(1) $1 \operatorname{RP}(\alpha ; u)$ is true for all $u(z)$ with $\left(1-|z|^{2}\right)^{n(\alpha-1)} u(z) \in C(\bar{B})$.

(2) $\alpha$ is real.

Proof of Lemma 2. (1) Suppose $g_{a_{1}}(r) \geq g_{a_{2}}(r)$ for some $r: 0<r<1$. Then since $g_{a_{1}}(r)<g_{a_{2}}(r)$ near $r=1$, there exists $r_{0}$ such that $g_{a_{1}}\left(r_{0}\right)=g_{a_{2}}\left(r_{0}\right)$. This $g_{a_{1}}$ and $r_{0}$ satisfy the condition of Theorem 1 with $\alpha=a_{2}, r(z)=r_{0}$, and $u=g_{a_{1}}$, but $g_{a_{1}} \notin X_{\lambda_{a}}$. This is a contradiction.

(2) Let $\beta=b+i c, c \neq 0$, be fixed. Let $\mathscr{B}(r)=g_{\beta}(r) / g_{b}(r), 0 \leq r<1$, and let $R=\left\{(a, r): \frac{1}{2}<a<b\right.$ and $\left.0<r<1\right\}$. Define

$$
G(a ; r)=g_{a}(r) / g_{b}(r), \quad(a, r) \in \bar{R} .
$$

Since $g_{b}(r)=\left(1-r^{2}\right)^{n(1-b)} \mathscr{F}(b: r), \mathscr{F}(b: 0)=1$, and $\mathscr{F}(a: r)$ is increasing, it follows that $g_{b}(r)$ cannot be zero and that $G(a ; r)$ is well defined continuous 
in each variable. Note also, by $(1)$, that $G(a ; r)$ is strictly increasing in the first variable. Let $\Omega$ be the simply connected open region surrounded by

$$
\left\{G\left(\frac{1}{2} ; r\right): 0 \leq r \leq 1\right\} \cup\{(a, 1): 0 \leq a \leq 1\} \cup\{(1, r): 0 \leq r \leq 1\} .
$$

Then

$$
\Omega \subset \bigcup_{(a ; r) \in R}(r, G(a ; r))
$$

because if $(x, y) \in \Omega$ then $G\left(\frac{1}{2} ; x\right)<y<G(b ; x)=1$, and by use of the intermediate value theorem we can find $a: \frac{1}{2}<a<b$ such that $G(a ; x)=y$.

Next let $\mathscr{B}=\lim _{r \rightarrow 1}|\mathscr{B}(r)|$. We claim

$$
0<\mathscr{B}<1 \text {. }
$$

It is obvious that $\mathscr{B} \leq 1$. Also, since $|\mathscr{F}(\beta: r)| \leq \mathscr{F}(b: r) \uparrow \mathscr{F}(b)$, by the dominated convergence theorem and (3),

Since

$$
|\mathscr{B}(1)|=\lim _{r \rightarrow 1} \frac{\left|g_{\beta}(r)\right|}{g_{b}(r)}=\frac{\lim _{r \rightarrow 1}|\mathscr{F}(\beta: r)|}{\lim _{r \rightarrow 1} \mathscr{F}(b: r)}=\frac{|\mathscr{F}(\beta: 1)|}{\mathscr{F}(b)} .
$$

$$
\begin{aligned}
\mathscr{F}(\beta: 1) & =\lim _{r \rightarrow 1} \mathscr{F}(\beta: r)=\lim _{r \rightarrow 1} F\left(n-n \beta, n-n \beta, n: r^{2}\right) \\
& =\frac{\Gamma(n) \Gamma(2 n \beta-n)}{\Gamma^{2}(n \beta)},
\end{aligned}
$$

where $F(\cdots)$ is the Gaussian hypergeometric function (see [3] or [4, p. 54]), we have $\mathscr{B} \neq 0$. Suppose $|\mathscr{F}(\beta: 1)|=\mathscr{F}(b)$. Then $\gamma \mathscr{F}(\beta: 1)=\mathscr{F}(b)$ for some $\gamma$ with $|\gamma|=1$, that is,

$$
\int_{S}\left(\frac{1}{\left|1-\zeta_{1}\right|^{2 n(1-b)}}-\frac{\gamma}{\left|1-\zeta_{1}\right|^{2 n(1-\beta)}}\right) d \sigma(\zeta)=0,
$$

from which follows $1=\operatorname{Re}\left(\gamma\left|1-\zeta_{1}\right|^{i 2 n c}\right)$ a.e., $\zeta \in S$. This is impossible.

Now, since $\mathscr{F}(\beta: r) / \mathscr{F}(b: r)$ has a nonzero finite limit as $r \rightarrow 1$,

$$
\mathscr{B}(r)=\left(1-r^{2}\right)^{-i n c} \frac{\mathscr{F}(\beta: r)}{\mathscr{F}(b: r)}
$$

makes a curve that winds the origin infinitely many times as $r$ approaches to 1. In particular, we can take, by $(12)$, a positive sequence $\mathscr{B}\left(r_{j}\right)$ such that $1>\mathscr{B}\left(r_{j}\right) \rightarrow \mathscr{B}>0$ as $r_{j} \rightarrow 1$. Recalling (11), we can conclude that there are infinitely many $r_{j}$ such that

$$
\left(r_{j}, \mathscr{B}\left(r_{j}\right)\right) \subset \Omega \subset \bigcup(r, G(a ; r)) .
$$

Therefore there are $a$ and $r_{j}$ such that $\mathscr{B}\left(r_{j}\right)=G\left(a ; r_{j}\right)$, that is, $g_{\beta}\left(r_{j}\right)=$ $g_{a}\left(r_{j}\right)$.

Proof of Theorem 3. That (2) $\Rightarrow$ (1) follows from Theorem 1. For the converse, first fix a nonreal $\beta$ with $\operatorname{Re} \beta>\frac{1}{2}$. Take $a$ and $r$ as in Lemma 2(2). Then by (ii),

and by (iii) and (2),

$$
g_{a}(z)\left(1-|z|^{2}\right)^{n(\beta-1)} \in C(\bar{B}),
$$

$$
g_{a}(z) g_{\beta}(r)=\int_{S} g_{a}\left(\phi_{z}(r \zeta)\right) d \sigma(\zeta),
$$

but $g_{a}(z) \in X_{\lambda_{a}}$ (so that $\left.g_{a}(z) \notin X_{\lambda_{\beta}}\right)$. Therefore $1 \mathrm{RP}\left(\beta ; g_{a}\right)$ is false. 


\section{THe CASE $\alpha=\frac{1}{2}$}

When $\alpha=\frac{1}{2}$, we have a stronger result as expected in the corollary to Theorem 1.

Theorem 4. Suppose that

$$
u(z)=\left(1-|z|^{2}\right)^{n / 2} \frac{1}{|z|^{2}} \log \frac{1}{1-|z|^{2}} F(z)
$$

for some $F \in C(\bar{B})$. If for every $z \in B$ there corresponds an $r(z): 0<r(z)<1$ and an automorphism $\psi_{z}$ such that (4) holds, then

$$
u(z)=\frac{1}{\mathscr{G}(1 / 2)} \int_{S} P^{1 / 2}(z, \zeta) F(\zeta) d \sigma(\zeta), \quad z \in B .
$$

In particular, $1 \mathrm{RP}\left(\frac{1}{2} ; u\right)$ is true.

Proof of Theorem 4. The idea is easier than, but almost the same as, that of the proof of Theorem 1. Let $F^{\prime}(z)=F(z) / \mathscr{G}\left(\frac{1}{2}:|z|\right)$. Then by Lemma 1 $F^{\prime} \in C(\bar{B})$ also. Let

$$
v(z)=\int_{S} P^{1 / 2}(z, \zeta) F^{\prime}(\zeta) d \sigma(\zeta),
$$

and

$$
G(z)= \begin{cases}g_{1 / 2}(z)^{-1} v(z), & z \in B, \\ F^{\prime}(z), & z \in S .\end{cases}
$$

Then since $\left(1-r^{2}\right)^{n / 2} g_{1 / 2}(r)^{-1} \rightarrow 0$ as $r \rightarrow 1$, it follows that $G(r \zeta) \rightarrow G(\zeta)$ uniformly on $\zeta \in S$ as $r \rightarrow 1$. Hence $G \in C(\bar{B})$. Now let $h(z)=u(z)-v(z)$. It suffices to show that the function

$$
H(z)= \begin{cases}g_{1 / 2}(z)^{-1} h(z), & z \in B, \\ 0, & z \in S,\end{cases}
$$

is identically zero in $B . H \in C(\bar{B})$ by Lemma 1 . Suppose $|H(z)|>0$ for some $z \in B$. Let $E$ be the set on which $|H(z)|$ takes its maximum, and let $z_{0} \in E$ be such that $\operatorname{dist}\left(z_{0}, S\right)=\operatorname{dist}(E, S)$. Then by (4)

$$
\begin{aligned}
H(z) g_{1 / 2}(r(z)) & =\int_{S} g_{1 / 2}(z)^{-1} h \circ \psi_{z}(r(z) \zeta) d \sigma(\zeta) \\
& =\int_{S} \frac{g_{1 / 2}\left(\psi_{z}(r \zeta)\right)}{g_{1 / 2}(z)} H \circ \psi_{z}(r \zeta) d \sigma(\zeta) .
\end{aligned}
$$

If we apply $z_{0}$ with its corresponding $r\left(z_{0}\right)=r_{0}$, we obtain, by (2) and by the location of $z_{0}$,

$$
\left|H\left(z_{0}\right)\right| g_{1 / 2}\left(r_{0}\right)<\left|H\left(z_{0}\right)\right| g_{1 / 2}\left(r_{0}\right),
$$

which is a contradiction. 
The growth condition in Theorem 4 is sharp in the sense that there is $u(z)$ such that $F(z)$ is bounded on $B$ and $1 \operatorname{RP}\left(\frac{1}{2} ; u\right)$ is false. We can see this by following exactly the same lines of the proof of Theorem 2 .

\section{ACKNOWLEDGMENT}

This work was completed while the author visited the University of Wisconsin-Madison. The author would like to thank Professor Patrick Ahern for helpful discussions.

\section{REFERENCES}

1. K. Izuchi, The one radius theorem is not true for bounded real analytic functions, Proc. Amer. Math. Soc. 130 (1988), 823-830.

2. Oliver D. Kellog, Converses of Gauss's theorem of the arithmetic mean, Trans. Amer. Math. Soc. 30 (1934), 227-242.

3. Lucy John Slater, Generalized hypergeometric functions, Cambridge Univ. Press, London and New York, 1966.

4. Walter Rudin, Function theory in the unit ball of $\mathbf{C}^{n}$, Springer-Verlag, New York, 1980.

Department of Mathematics Education, Andong National University, Andong KYUNGPOOK 760-749, KOREA 